\title{
Envolvimento, desempenho acadêmico e composição escrita
}

\author{
Maria Isabel Festas ${ }^{1}$ \\ Maria José Prata ${ }^{1}$ \\ Albertina Lima de Oliveira ${ }^{1}$ \\ Feliciano Veiga ${ }^{2}$
}

\section{Resumo}

O envolvimento pode ser considerado um fator decisivo na manutenção da participação dos alunos na sua aprendizagem, sendo isso particularmente relevante quando se trata de áreas muito exigentes, como a composição escrita. É assim que a relação entre o envolvimento e o desempenho acadêmico tem sido amplamente documentada, embora o caso da escrita tenha sido menos estudado. No presente trabalho, procurou-se saber se o envolvimento escolar se relaciona com o desempenho acadêmico e com a composição escrita. Através de um estudo correlacional, 213 alunos do $9^{\circ}$ ano de escolaridade responderam ao Questionário de Envolvimento dos Alunos na Escola, que inclui as dimensões comportamental, afetiva, cognitiva e agenciativa. Os mesmos alunos escreveram um texto argumentativo que foi avaliado do ponto de vista da sua qualidade global e da sua estrutura. Recolheram-se, ainda, as notas obtidas em várias disciplinas e dados relativos às repetências. Pelo estabelecimento de correlações, verificou-se que o envolvimento está associado ao rendimento escolar e à escrita, mas, neste último caso, apenas à sua qualidade global. Constatou-se, também, que as diferentes dimensões do envolvimento não se relacionam de igual modo com o desempenho acadêmico e com a escrita, destacando-se a ausência de uma associação com a componente agenciativa. Sendo possível concluir-se pela existência de uma relação entre envolvimento e desempenho acadêmico e escrita, apontam-se algumas das principais limitações do presente estudo e indicam-se linhas de investigação futura que ajudem a perceber alguns dos resultados encontrados.

\section{Palavras-chave}

Envolvimento na escola - Composição escrita - Desempenho acadêmico.

1- Universidade de Coimbra, Portugal. Contatos: ifestas@fpce.uc.pt; mprata58@gmail.com; aolima@fpce.uc.pt

2- Universidade de Lisboa, Portugal. Contato: fhv@ie.ulisboa.pt 


\section{Engagement, academic achievement and written composition}

\section{Abstract}

Engagement can be considered as a crucial factor in maintaining student participation in their own learning, mainly in demanding areas such as written composition. It is this way that the relationship between engagement and academic achievement has been broadly documented, although the case of writing has been less studied. This study intended to investigate if engagement is related to academic achievement and written composition. Through a correlational study, 213 ninth-graders answered to the Questionnaire on Students Engagement in School, which includes behavioral, affective, cognitive, and agentic dimensions. The same students wrote an argumentative text that was evaluated from the point of view of its global quality and structure. Data related to students' grades in different subject areas and students' retentions were also collected. By establishing correlations, it was observed that engagement is associated with academic achievement and also with writing, but only with global quality of writing. It was also found that the different dimensions of engagement were unequally related to academic achievement and writing, being particularly noticeable the absence of an association with agentic dimension. Conclusion was the existence of a relationship between engagement and academic achievement and writing, some of the main limitations of the study were indicated as well as lines of future research that would help understand some of the results found.

\section{Keywords}

Engagement in school - Written composition - Academic achievement.

\section{Introdução}

0 envolvimento tem sido conceituado como o grau de motivação e comprometimento com a escola e com a aprendizagem acadêmica (SIMON-MORTON; CHEN, 2009). Tendo uma natureza multifacetada, o envolvimento inclui as dimensões comportamental, afetiva, cognitiva e agenciativa (VEIGA et al., 2012). Embora os autores se diferenciem quanto ao papel que atribuem a cada uma destas dimensões, há, atualmente, um largo consenso em torno da importância do envolvimento, admitindo-se que se trata de um fator estreitamente relacionado com o desempenho acadêmico, sendo, em simultâneo, preventivo de muitos problemas como o abandono escolar e a delinquência juvenil (e.g., APPLETON; CHRISTENSON; FURLONG, 2008; REEVE; TSENG, 2011).

Concebido como fundamental na manutenção da participação dos alunos no processo da sua aprendizagem, o envolvimento torna-se decisivo em tarefas exigentes como são 
as escolares. Nesse sentido, a composição escrita, área transversal a toda a aprendizagem, assume um lugar de destaque, já que requer a mestria de competências múltiplas relativas à ortografia, ao léxico, às regras de pontuação, sintáticas, morfológicas e de construção textual, implicando, igualmente, um grande domínio de estratégias de resolução de problemas, como a planificação e a revisão (cf. KELLOGG, 1994). Dada a complexidade da escrita, facilmente se entende a importância dos aspectos motivacionais e, em particular, do envolvimento, que será um elemento essencial para manter a persistência necessária à prossecução de uma tarefa desta natureza (cf. BOSCOLO; GELATI, 2007; BRUNING; KAUFFMAN, 2016). Assistindo-se, desde há alguns anos, a um interesse crescente no papel da motivação na escrita, é desejável que esse interesse se estenda ao envolvimento, aspecto ainda pouco estudado nessa área acadêmica específica (cf. BOSCOLO; HIDI, 2007; HIDI; BOSCOLO, 2007).

É neste contexto que, recorrendo ao Questionário de Envolvimento dos Alunos na Escola (VEIGA, 2013; 2016), o presente trabalho tem como objetivo fundamental saber se, efetivamente, o envolvimento se relaciona com o desempenho acadêmico e com o nível de realização da composição escrita de alunos do $9^{\circ}$ ano de escolaridade. Uma vez que o questionário usado dá indicações sobre as componentes comportamental, afetiva, cognitiva e agenciativa, pretende-se, igualmente, perceber não apenas se o desempenho acadêmico e a eficiência na composição escrita se relacionam com o envolvimento global, mas ainda como é que nessa relação se diferenciam ou não as quatro dimensões.

Após uma breve resenha sobre o envolvimento e suas relações com o desempenho acadêmico e com a escrita, é apresentado o estudo de natureza correlacional levado a cabo em escolas públicas portuguesas, descrevendo-se os participantes, instrumentos, procedimento e resultados. Por último, é feita uma discussão dos resultados alcançados, apresentam-se algumas conclusões, bem como as principais limitações deste estudo e sugestões de investigações futuras.

\section{Envolvimento dos alunos na escola}

0 envolvimento está estreitamente ligado à motivação, havendo autores como Anderman e Patrick (2012) que o fazem depender dos motivos, tal como são definidos na Teoria dos Objetivos de Elliot (1999) - objetivos de mestria/competência/aprendizagem e de desempenho. Segundo essa perspectiva, a orientação para a mestria está associada a comportamentos acadêmicos positivos, à participação em atividades fora da escola e à ausência de comportamentos disruptivos. Os objetivos de incentivo adotados pelas pessoas, influenciados pelo valor subjetivo atribuído à tarefa, contribuirão, assim, para o seu envolvimento nesta mesma tarefa (ECCLES et al., 1993; WIGFIELD; ECCLES, 2000). Sendo a motivação uma condição fundamental, não é, contudo, suficiente, pois só através do envolvimento se torna possível uma participação efetiva na aprendizagem (SKINNER et al., 2009). Efetivamente, o envolvimento é concebido como sendo o responsável pela iniciação e continuidade das ações necessárias à realização das tarefas.

Existe um alargado consenso quanto à natureza multidimensional do envolvimento, que inclui de duas a quatro dimensões. 0 modelo dual considera a dimensões afetiva ou 
emocional e a comportamental (FINN, 1989; SKINNER et al., 2009), enquanto o modelo tripartido propõe, para além das duas anteriores, a dimensão cognitiva (FREDRICKS; BLUMENFELD; PARIS, 2004). Mais recentemente, alguns autores introduziram uma nova componente, a agenciativa (REEVE; TSENG, 2011).

A dimensão afetiva, emocional ou psicológica (APPLETON; CHRISTENSON; FURLONG, 2008; GLANVILLE; WILDHAGEN, 2007) diz respeito às atitudes, ao sentido de identificação e de pertença dos alunos à escola, à aprendizagem, aos colegas e aos professores. A vivência da escola como uma experiência satisfatória e agradável e o interesse por aquilo que nela se passa fazem parte dessa dimensão (GOODENOW, 1993; JOHNSON; CROSNOE; ELDER, 2001; SKINNER; BELMONT, 1993; VOELKL, 1997).

A dimensão comportamental inclui as práticas e os comportamentos vocacionados para a aprendizagem, levados a cabo em casa, na turma e na escola. A realização dos trabalhos de casa traduz o empenho que, fora da escola, os alunos colocam nas tarefas acadêmicas (SHARP; KEYS; BENEFIELD, 2001). A participação nas atividades da turma, através dos trabalhos de grupo, da atenção e da discussão nas aulas, e a ausência de absentismo denotam o envolvimento comportamental dos alunos na classe e na escola (REID, 2005; SKINNER et al., 2009). A obtenção de boas notas, a participação em atividades extracurriculares, a ausência de comportamentos disruptivos e a aceitação e o respeito das regras da escola também são aspectos considerados como integrantes dessa dimensão (cf. VEIGA et al., 2012).

A componente cognitiva reporta-se às crenças dos alunos acerca de si próprios e da sua competência acadêmica e às suas percepções da escola, dos professores e dos colegas (JIMERSON; CAMPOS; GREEN, 2003; MARTIN, 2007). Esta dimensão inclui, igualmente, o investimento do estudante, bem como o recurso a estratégias de autorregulação necessárias à realização de tarefas intelectualmente complexas e exigentes (APPLETON et al., 2006; FREDRICKS; BLUMENFELD; PARIS, 2004). Nesse sentido, a componente cognitiva dá conta do grau de profundidade do envolvimento do estudante na sua aprendizagem e nas tarefas acadêmicas.

A dimensão agenciativa, relacionada com a iniciativa e a ação por parte dos alunos, tem sido evidenciada, por investigações recentes (REEVE; TSENG, 2011), como sendo uma componente importante do envolvimento na escola. Trata-se da capacidade dos alunos de intervir e influenciar o meio escolar e o seu processo de aprendizagem, contribuindo de forma proativa e construtiva para o desenvolvimento da instrução que recebem e para o enriquecimento das condições acadêmicas.

\section{Envolvimento e desempenho acadêmico}

0 envolvimento pode ser um importante mediador entre variáveis pessoais (por exemplo, de ordem motivacional, percepções) e contextuais (por exemplo, tipo de recursos existentes), por um lado, e o desempenho acadêmico, por outro (LI; LERNER; LERNER, 2010; WANG; HOLCOMBE, 2010). Estudos longitudinais, como os de Voelkl (1997), em que se seguiram alunos do $4^{\circ}$ ao $8^{\circ}$ ano, têm mostrado que a relação entre envolvimento e desempenho escolar se mantém ao longo da escolaridade, embora se saiba que o primeiro 
vai diminuindo dos primeiros para os últimos anos passados na escola (KLEM; CONNELL, 2004). As relações entre desempenho acadêmico e envolvimento não serão, certamente, alheias à influência do meio sociocultural. Alunos de ambientes mais desfavorecidos apresentam visões mais negativas de si próprios, da escola e da carreira (BORKOWSKI; THORPE, 1994).

Estudando a influência das várias dimensões do envolvimento na realização acadêmica, autores como Finn (1989) consideram que a comportamental, através da participação na escola e nas atividades da classe, conduzindo a uma melhor realização e a uma maior competência, ajudará o aluno a desenvolver o sentido de identificação com a escola, contribuindo, assim, para um aumento do envolvimento afetivo. Já outros autores como Skinner et al. (2008) consideram que é o envolvimento afetivo que determinará o desempenho, sendo esta relação mediada pela dimensão comportamental. Nesta perspectiva, alunos com atitudes mais favoráveis em relação à escola participariam mais das atividades da turma e dos trabalhos de casa, tendo, assim, um maior aproveitamento escolar. Do mesmo modo, atitudes negativas para com a escola conduziriam a um maior absentismo.

Quer se dê maior relevância a uma ou a outra dimensão, há atualmente forte evidência das relações entre, por um lado, o envolvimento, e, por outro, o desempenho acadêmico (LI et al., 2008), a aprendizagem (AINLEY, 1993), as notas escolares e os resultados em testes estandardizados (CARAWAY et al., 2003).

Sendo o envolvimento um fator muito importante nos bons desempenhos acadêmicos, sabe-se que a sua ausência está associada ao insucesso e abandono escolares. Comparando alunos com diferentes trajetórias desenvolvimentais, Janosz e colaboradores (2008) verificaram que aqueles que, entre os 12 e os 16 anos, mantêm um envolvimento elevado têm uma ocorrência mínima de abandono, contrariamente aos que, nessas mesmas idades, diminuem os níveis de envolvimento e que apresentam uma maior probabilidade de deixar a escola. Também em relação à delinquência e ao uso de substâncias, tem-se encontrado dados que apontam no mesmo sentido, isto é, o de uma associação entre os padrões de desenvolvimento - mesmos níveis ou decréscimo de envolvimento - e, respectivamente, percursos normativos e problemáticos (HENRY; KNIGHT; THORNBERRY, 2012). Na verdade, muitos estudos têm mostrado que os problemas de comportamento (FREDRICKS; BLUMENFELD; PARIS, 2004), a delinquência (PAYNE, GOTTFREDSON; GOTTFREDSON, 2003) e o consumo de substâncias (GUTMAN; MIDGLEY, 2000), todos eles fortemente ligados a um fraco desempenho acadêmico, estão relacionados com baixos níveis de envolvimento escolar. Há dados particulares relativos a algumas das dimensões do envolvimento que indicam que a emocional e a comportamental são boas preditoras de uma ausência ou decréscimo da delinquência e do uso de substâncias (LI; LERNER, 2011) e que, do mesmo modo, a cognitiva se relaciona negativamente com os comportamentos disruptivos (HIRSCHFIELD; GASPER, 2011).

\section{Envolvimento e escrita}

Escrever requer não apenas o domínio de competências relacionadas com ortografia, pontuação, construção frásica, estilo e estruturas textuais, mas também a processos de 
procura de informação, planificação, tradução e revisão, tal como foi salientado há já alguns anos por Hayes e Flower (1980). Equiparada, por esses autores, a uma situação de resolução de problemas, a escrita exige, no momento da planificação, o estabelecimento de objetivos a que se deve subordinar a escolha das ações a empreender, bem como a revisão de acordo com o que foi previamente definido. A escrita madura, pressupondo uma transformação dos conhecimentos que se procuraram interna ou externamente em algo de mais elaborado e/ou pessoal (SCARDAMALIA; BEREITER, 1986), constitui um meio privilegiado de construção de conhecimento e de aprendizagem (cf. MACARTHUR; GRAHAM, 2016). Trata-se, assim, de uma tarefa muito importante, mas também muito exigente, o que estará na origem das dificuldades generalizadas encontradas em alunos de todos os níveis de escolaridade (por exemplo, GABINETE DE AVALIAÇÃO EDUCACIONAL, 2013; NATIONAL CENTER OF EDUCATIONAL STATISTICS, 2012), ficando os resultados muito aquém do que seria de esperar (LANE et al., 2006).

Dada a complexidade da escrita, facilmente se compreende a necessidade de um envolvimento por parte do aluno. Efetivamente, a composição textual exige esforço, prática e comprometimento, não resultando de uma atividade acidental. Para que alguém mantenha a sua persistência, dedicando o tempo e a energia necessários à resolução de uma tarefa desse tipo, precisa estar motivado e se sentir envolvido nessa mesma tarefa. No entanto, apesar de nos últimos anos as ligações da escrita com a motivação terem suscitado um grande interesse (cf., BRUNING; KAUFFMAN, 2016), o papel do envolvimento nesse domínio específico não tem sido objeto de muita atenção. A relação do envolvimento com uma abordagem comprometida com a aprendizagem foi já documentada em áreas de literacia, como a leitura e a compreensão de textos (GUTHRIE; WIGIELD, 2000), sendo de esperar que isso mesmo aconteça com a escrita (por exemplo, BOSCOLO; HIDI, 2007). De fato, tendo Guthrie e Wigield (2000) verificado que os alunos que mais se envolvem são aqueles que leem de forma mais ativa, usando estratégias que lhes permitem adquirir conhecimentos avançados e níveis de compreensão mais profundos, é expectável que na escrita, tarefa complexa e de literacia como a da leitura, o envolvimento esteja, igualmente, associado a um investimento e esforço e a uma maior capacidade para recorrer e gerir os processos necessários à sua boa prossecução, traduzindo-se em melhores desempenhos e em composições de boa qualidade.

\section{Método}

Nesta secção apresentam-se a caracterização da amostra, o procedimento seguido, desde as solicitações para a autorização do estudo até à recolha dos dados empíricos, bem com os instrumentos de avaliação utilizados.

\section{Participantes}

Participaram no estudo 213 alunos com idades compreendidas entre os 14 e os 18 anos $(M=14,56 ; D P=0,70)$, tendo maioritariamente 14 anos $(n=112)$, seguidos por 15 anos $(n=79)$ e por 16 ou mais anos $(n=22)$. Destes, $111(52,1 \%)$ pertencem ao sexo masculino e 102 (47,9\%) ao sexo feminino. 
Os alunos encontravam-se a frequentar o $9^{\circ}$ ano de escolaridade em três escolas da zona central de Portugal, distribuídos por doze turmas. Nestas, o número de alunos variava entre 14 e 22. Quanto às habilitações literárias dos pais, consideraram-se três níveis de escolaridade - básico (até ao $9^{\circ}$ ano), secundário (até ao $12^{\circ}$ ano) e superior -, sendo que, no caso do pai, 89 apresentavam uma formação até ao $9^{\circ}$ ano (41,8\%), 59 até ao $12^{\circ}$ ano $(27,7 \%)$ e $51(23,9 \%)$ tinham uma formação superior. No caso da mãe, 75 $(35,2 \%)$ tinham o nível básico, 57 (26,8\%) o nível secundário e $71(33,3 \%)$ o nível superior.

\section{Procedimentos e instrumentos}

Antes de o estudo começar, foram pedidas autorizações e obtido consentimento da Direção Geral do Ensino Básico (DGE), dos diretores das escolas, dos professores, dos encarregados de educação e dos alunos. Na recolha de dados foram respeitados todos os requisitos éticos necessários a este tipo de investigação.

0 envolvimento dos alunos foi avaliado através do Questionário de Envolvimento dos Alunos na Escola - Uma Escala com Quatro Dimensões EAE - E4D (VEIGA, 2013; 2016). Trata-se de um instrumento com vinte questões que contemplam as quatro dimensões do envolvimento: cognitiva, afetiva, comportamental e agenciativa. A dimensão cognitiva comporta cinco questões (itens 1 a 5) relacionadas com o investimento do aluno no processamento de informação e com estratégias de autorregulação (por exemplo: Procuro relacionar o que aprendo numa disciplina com o que aprendi noutras; Revejo regularmente os meus apontamentos, mesmo que um teste ainda não esteja próximo), incluindo um item de escrita (Quando escrevo os meus trabalhos, começo por fazer um plano para $o$ texto a redigir). Os itens da dimensão afetiva (itens 6 a 10) incidem na ligação e no sentido de pertença à escola (por exemplo: A minha escola é um lugar onde me sinto integrado; A minha escola é um lugar onde faço amigos com facilidade). Na dimensão comportamental, os seus cinco itens (11 a 15) avaliam aspectos ligados a condutas nas aulas e na escola (por exemplo: Perturbo a aula propositadamente; Falto à escola sem uma razão válida). Por último, a dimensão agenciativa inclui igualmente cinco itens (16 a 20) que se relacionam com a contribuição construtiva do aluno para a sua aprendizagem, visando às suas iniciativas, intervenções, questões e sugestões (por exemplo: Falo com os meus professores sobre aquilo de que gosto e não gosto; Durante as aulas, coloco questões aos professores). Para além das quatro dimensões, o somatório do EAE - E4D dá-nos uma medida geral do envolvimento do aluno na escola.

0 EAE - E4D é uma escala de tipo Likert, com 6 pontos, os quais variam entre 1 (total desacordo) e 6 (total acordo). A maioria dos itens estão formulados de modo positivo, embora alguns estejam em sentido contrário (com os resultados mais baixos a indicarem maior envolvimento). Trata-se de uma escala que revelou boas propriedades psicométricas, quer em termos de validade, quer de fidelidade. Os valores globais obtidos na presente amostra variaram entre 55 e 114 e revelaram um $\alpha$ de Cronbach de .80. No que respeita à dimensão cognitiva, os valores situaram-se entre 9 e 27, e a sua consistência interna foi de .67. No caso da dimensão afetiva, encontraram-se valores entre 12 e 30, e a consistência interna obtida foi de .78. Quanto à dimensão comportamental, a amplitude 
dos valores situou-se entre 10 e 30, com um $\alpha$ de Cronbach de .89 e, no que respeita à dimensão agenciativa, os valores variaram entre 5 e 30, sendo o $\alpha$ de Cronbach de .87 .

Tendo em vista a avaliação do desempenho acadêmico, uma bolseira de investigação recolheu, nos registos escolares dos alunos, as notas obtidas no último período de aulas do ano letivo anterior, nas disciplinas de português, língua estrangeira, história, matemática, ciências naturais, ciências físicas e química e geografia. Foi feita uma média das notas de todas as disciplinas, com exceção de português, que foi considerada independentemente das outras, já que, tratando-se da disciplina de língua materna, se relaciona estreitamente com a escrita, área que interessa particularmente no contexto do presente trabalho. Daqui resultaram dois indicadores dos resultados escolares: a nota de português e a média das notas do conjunto das outras disciplinas. Considerou-se, ainda, o número de repetências apresentado por cada aluno.

Com o objetivo de avaliar a escrita, foi pedido a todos os alunos que redigissem um texto argumentativo sobre o tema $O$ papel do grupo de amigos, suas vantagens $e$ desvantagens. Esse tema foi escolhido por sugestão dos professores, que o indicaram como sendo de especial interesse para os alunos dessas idades. As composições foram escritas em sala de aula, no âmbito da disciplina de português.

Depois de ter sido retirada a identificação dos alunos, procedeu-se à avaliação dos textos. Uma medida da sua qualidade global foi obtida a partir da classificação feita por dois professores de português. Estes, de forma independente, atribuíram um valor, numa escala de 0 a 5, à qualidade de cada composição, atendendo a critérios como organização das ideias, construção frásica, respeito pela gramática e vocabulário. A fidelidade inter-cotadores (Kappa de Cohen) foi de .76.

Tratando-se de um texto argumentativo, foi, também, avaliada a estrutura das composições. Assim, foi contabilizado o número de elementos estruturais característicos da argumentação presentes em cada texto: introdução, tomada de posição, argumentos a favor, contra-argumentos e conclusão (cf. VAN EEMEREN; GROOTENDORST, 2004). Foram atribuídos 0 e 1 pontos, respectivamente, à ausência e à presença de cada um dos seguintes elementos: introdução, tomada de posição e conclusão. Por sua vez, cada argumento e contra-argumento referido no texto era cotado com 1 ponto. 0 somatório de todos os pontos deu uma medida relativa à estrutura do texto. Dois assistentes de investigação, previamente treinados, avaliaram, de forma independente, todas as composições do ponto de vista da sua estrutura. A fidelidade inter-cotadores (Kappa de Cohen) foi de .95.

\section{Resultados}

Os dados foram tratados com recurso ao programa IBM SPSS, versão 22.

A maioria dos alunos, correspondendo a $84,5 \%(n=180)$ não apresentava repetências, 19 reprovaram uma vez (8,9\%), 13 duas vezes $(6,1 \%)$ e um $(0,5 \%)$ não respondeu.

A Tabela 1 apresenta os valores mínimos e máximos, a média, mediana e desvio padrão do envolvimento dos alunos na escola, do rendimento escolar, avaliado através da nota na disciplina de português e da média das notas do conjunto das outras disciplinas (língua estrangeira, história, matemática, ciências naturais, ciências físicas e química e 
geografia) e da classificação obtida na composição escrita, quer do ponto de vista da sua qualidade global, quer da sua estrutura.

Tabela 1 - Valores mínimos e máximos, média, mediana e desvio padrão das variáveis em estudo

\begin{tabular}{|c|c|c|c|c|c|c|}
\hline Variáveis & $N$ & Min. & Máx. & Média & Mediana & $D P$ \\
\hline Envolvimento Geral & 213 & 55.00 & 114.00 & 87.62 & 88.00 & 11.05 \\
\hline D. Cognitiva & 213 & 9.00 & 27.00 & 19.21 & 19.00 & 3.92 \\
\hline D. Afetiva & 213 & 12.00 & 30.00 & 25.04 & 26.00 & 4.21 \\
\hline D. Comportamental & 213 & 10.00 & 30.00 & 25.69 & 27.00 & 4.69 \\
\hline D. Agenciativa & 213 & 5.00 & 30.00 & 17.68 & 18.00 & 5.37 \\
\hline RE Português & 213 & 2.00 & 5.00 & 3.33 & 3.00 & 0.78 \\
\hline Média das Notas & 213 & 2.00 & 5.00 & 3.44 & 3.29 & 0.65 \\
\hline Q. Global Escrita & 213 & 0.00 & 4.00 & 1.66 & 1.50 & 0.76 \\
\hline Estrutura Escrita & 213 & 0.00 & 11.00 & 1.44 & 1.00 & 2.19 \\
\hline
\end{tabular}

Min.= Valor mínimo; Máx.= Valor máximo; DP= Desvio padrão; D= Dimensão; $\mathrm{RE}=$ Rendimento Escolar; Q= Qualidade.

Fonte: dados da pesquisa.

Na Tabela 1 incluímos a mediana e não apenas a média, como medidas de tendência central, porque alguns resultados estavam muito concentrados em torno de um ou dois valores, sendo o caso da estrutura da escrita, que embora variasse entre 0 e 11 , revelou que a grande maioria dos alunos apresentou pontuações muito baixas (82,1\% com 0 e 1 pontos), sem grande variabilidade nos outros resultados (3,4\% com 2 e 3 pontos, 6,5\% com 4 e 5 pontos, 3,7\% com 6 e 7 pontos, 2,9\% com 8 e 9 pontos, 1,4\% com 10 e 11 pontos).

Na Tabela 2 apresentam-se as correlações encontradas entre o envolvimento geral e as suas diferentes dimensões, por um lado, e, por outro, o desempenho na escrita (qualidade global e respeito pela estrutura argumentativa), os resultados escolares (nota de português e média das notas nas outras disciplinas), número de repetências e a idade.

Tabela 2- Correlações do envolvimento na escola com as outras variáveis

\begin{tabular}{|c|c|c|c|c|c|}
\hline & $\begin{array}{c}\text { Envolvimento } \\
\text { Geral }\end{array}$ & $\begin{array}{c}\text { D. } \\
\text { Cognitiva }\end{array}$ & $\begin{array}{c}\text { D. } \\
\text { Afetiva }\end{array}$ & $\begin{array}{c}\text { D. } \\
\text { Comportamental }\end{array}$ & $\begin{array}{c}\text { D. } \\
\text { Agenciativa }\end{array}$ \\
\hline Q. Global & $.173^{*}$ & $.270^{* *}$ & .028 & .008 & .129 \\
\hline Estrutura Escrita & -.071 & -.047 & -.034 & .001 & -.087 \\
\hline RE Português & $.247^{\star *}$ & $.143^{*}$ & .107 & $.270^{\star *}$ & .084 \\
\hline Média das Notas & $.320^{* *}$ & $.168^{*}$ & $.192^{\star}$ & $.339^{* *}$ & .090 \\
\hline$N^{0}$ repetências & $-.210^{\star \star}$ & -.041 & $-.181^{\star \star}$ & $-.271^{\star \star}$ & .112 \\
\hline Idade & $-.164^{*}$ & -.031 & $-.146^{\star}$ & $-.306^{\star *}$ & .066 \\
\hline
\end{tabular}

*Correlação significativa a nível 0.05 (bidirecional); ${ }^{* \star}$ Correlação significativa a nível 0.01 (bidirecional).

Fonte: dados da pesquisa. 
A partir da Tabela 2 pode-se constatar que a qualidade global da escrita se encontra significativamente associada ao envolvimento geral $(r=.173)$ e particularmente à dimensão cognitiva $(r=.270)$, em que a magnitude é moderada ${ }^{3}$ (NEWTON; RUDESTAM, 2013). No caso da estrutura, não se verifica qualquer correlação significativa. Quanto ao rendimento em português, para além da relação significativa com o envolvimento geral $(r=.247)$, são as dimensões cognitiva $(r=.143)$ e comportamental $(r=.270)$ que revelam uma associação significativa, sendo a magnitude desta última moderada. Tratando-se da média das notas dos alunos, todas as dimensões se revelam significativas, exceto a agenciativa, destacando-se o envolvimento geral $(r=.320)$ e a dimensão comportamental $(r=.339)$, cujas magnitudes do efeito são moderadas. Analisando o número de repetências, as quais variaram entre 0, 1 ou 2, para além de se registar uma relação significativa, mas negativa, com o envolvimento geral ( $r=-.210)$, as dimensões afetiva $(r=-.181)$ e comportamental ( $r$ $=-.271$ ) surgem igualmente significativamente relacionadas, sendo a magnitude do efeito desta última moderada. No que respeita à idade, encontra-se significativamente associada ao envolvimento geral ( $r=-.164)$, estando implicadas sobretudo as dimensões afetiva ( $r=$ -.146) e comportamental ( $r=-.306$ ), atingindo este último caso uma magnitude moderada.

Testando-se as diferenças entre rapazes e raparigas, não se encontraram diferenças significativas no envolvimento geral $\left(t_{(211)}=-.086, p=.932\right)$, nem nas quatro respectivas dimensões. Não foi encontrada igualmente qualquer diferença significativa entre os sexos, a nível das classificações escolares (português: $t_{(211)}=-.177, p=.860$; outras disciplinas: $t_{(211)}=.688, p=.492$ ) ou da composição escrita (holística: $t_{(211)}=-1.123, p=.263$; estrutura: $\left.t_{(211)}=-1.536, p=.126\right)$.

Tendo em consideração que as habilitações literárias dos pais e mães da amostra em estudo eram bastante heterogêneas (de níveis básico, secundário e superior), procurou-se saber se existiam diferenças significativas no envolvimento em função desta variável, através do teste da ANOVA univariada. No caso dos pais, não foi encontrada qualquer diferença significativa entre os três níveis contemplados no estudo; já no caso das mães obtiveramse diferenças significativas entre o nível 1 (básico) e o nível 3 (superior) nas dimensões afetiva $\left[F_{(2,200)}=.507, p=.007\right]$ e comportamental $\left[F_{(2,200)}=.324, p=.041\right]$, favorecendo os de nível superior, significando que quanto mais elevado é o nível educacional da mãe maior é o envolvimento afetivo e comportamental dos filhos na escola.

\section{Discussão, limitações, investigações futuras e conclusões}

No presente trabalho procurou-se averiguar se existia uma relação entre o envolvimento dos alunos na escola e o desempenho acadêmico em geral e na composição de textos em particular. Os resultados alcançados permitem confirmar, em grande parte, a existência de uma tal associação.

0 desempenho acadêmico, avaliado pela média das notas obtidas nas diferentes disciplinas no último período do ano letivo anterior, está estreitamente relacionado

3- Segundo os critérios de Cohen (NEWTON; RUDESTAM, 2013), podem se consideradas relações fracas, moderadas e elevadas, respectivamente, as que têm magnitudes do efeito próxima de .10, próximas de .30 e em torno de .50 ou superiores. 
com o envolvimento, sobretudo com o geral e com a sua dimensão comportamental. Assim, tal como previsto na literatura revista neste artigo, a identificação com a escola, o investimento no processamento de informação, o recurso a estratégias de autorregulação e, acima de tudo, os comportamentos vocacionados para a aprendizagem são variáveis que, neste estudo, se encontram estreitamente relacionadas com bons resultados escolares. Também o desempenho em português está bastante associado à dimensão comportamental e, embora em menor grau, ao envolvimento geral e à componente cognitiva. A relação entre envolvimento e desempenho vê-se confirmada pela associação entre o primeiro e as repetências. Com efeito, encontraram-se correlações negativas, estatisticamente significativas, entre o envolvimento geral e as suas dimensões afetiva e comportamental, por um lado, e o número de reprovações dos alunos, por outro, salientando-se, mais uma vez, a dimensão comportamental. Quanto mais repetências, menor é o envolvimento. As relações negativas entre as mesmas variáveis (envolvimento geral e dimensões afetiva e comportamental) e a idade podem ser lidas como corroborando estes últimos dados, e não no sentido de confirmarem a ideia de que o envolvimento vai diminuindo à medida que se progride na escolaridade (KLEM; CONNELL, 2004), já que os alunos eram todos do $9^{\circ}$ ano.

No que respeita à escrita, o envolvimento geral e, com grande destaque, a sua componente cognitiva estão, também, significativamente ligados à qualidade global dos textos produzidos. Sendo a escrita uma tarefa altamente exigente do ponto de vista dos recursos mentais e das estratégias de autorregulação (cf., MACARTHUR; GRAHAM, 2016), é compreensível que o envolvimento cognitivo assuma um tal papel. Não foi, no entanto, encontrada relação entre a qualidade da escrita e as outras dimensões do envolvimento. Do mesmo modo, não foi detectada nenhuma ligação entre o envolvimento e o respeito pela estrutura textual, argumentativa neste caso. Sendo esta última uma componente essencial da composição, tal ausência causa estranheza. Mas uma análise atenta dos resultados mostra que, na realidade, os alunos tiveram muitas dificuldades em incluir nos seus textos os elementos estruturais da argumentação, tendo níveis de desempenho muito baixos e bastante homogêneos. Havendo cinco unidades (introdução, tomada de posição, argumentos a favor, contra-argumentos e conclusão) e podendo duas delas - argumentos a favor e contra-argumentos - estar representadas mais do que uma vez (um texto argumentativo bem escrito deve conter vários argumentos a favor e vários contra-argumentos), seria de esperar que os resultados se distribuíssem uniformemente, num intervalo que fosse, pelo menos, de 0 a 9 pontos (contando com os cinco elementos e mais três argumentos a favor e três contra-argumentos). Ora, como foi já referido, a grande maioria dos alunos (82,1\%) teve uma pontuação de 0 e 1 , podendo, desse modo, justificar-se a ausência de correlações entre as medidas.

Pode-se, então, afırmar que o envolvimento geral está associado ao rendimento escolar e à escrita, embora, neste último caso, de forma menos notória. Quanto às várias dimensões do envolvimento, salienta-se a comportamental, correlacionada com as notas de português, a média das outras notas, as repetências e a idade. A dimensão cognitiva foi a única que se revelou associada à escrita (qualidade global), mostrando-se também relacionada com o desempenho acadêmico, avaliado quer pela média das notas de um grupo alargado de disciplinas quer pela nota de português. A dimensão afetiva também se destacou, no que respeita à relação com o desempenho acadêmico (notas em várias 
disciplinas e número de repetências). Como já foi referido, nenhuma das dimensões se revelou correlacionada com a escrita, a nível da sua estrutura.

Relativamente à dimensão agenciativa, contrariamente ao esperado, nenhuma relação foi encontrada. Segundo os dados do presente estudo, esta dimensão não está associada nem ao desempenho acadêmico nem à produção escrita. Referindo-se à capacidade dos alunos para terem iniciativa e para participarem proativa e construtivamente no seu percurso acadêmico (REEVE; TSENG, 2011), é de estranhar que a dimensão agenciativa não esteja correlacionada com os resultados escolares.

A diferenciação do papel das dimensões do envolvimento em função das variáveis em estudo e a ausência de uma relação de qualquer uma destas últimas com a componente agenciativa levantam questões que devem ser discutidas e investigadas. Em primeiro lugar, é possível que o instrumento usado e os seus itens tenham tido alguma influência nos resultados. Basta lembrar que a dimensão cognitiva inclui uma questão sobre a escrita, podendo esta ter influenciado a relação que foi encontrada entre as duas variáveis (dimensão cognitiva e composição). É desejável que, no futuro, se recorra a outros meios de estudo do envolvimento e se cruzem os dados obtidos, de modo a verificar se os presentes se confirmam. Mas os resultados encontrados podem apontar para a existência de outro tipo de relações que urge aprofundar e esclarecer. 0 fato de apenas a vertente cognitiva estar relacionada com a escrita pode sugerir que há uma diferenciação na presença das dimensões do envolvimento em função de áreas acadêmicas específicas. Novos estudos são necessários para perceber se é realmente isso que acontece. As características da tarefa da escrita podem implicar uma relação mais estreita com a componente cognitiva, enquanto que as de outras áreas acadêmicas poderão remeter para ligações preferenciais com as dimensões afetiva, comportamental ou agenciativa. No futuro, será interessante explorar essas relações, avaliando, não apenas as que existem entre envolvimento e escrita, mas, igualmente, entre o primeiro e outros domínios acadêmicos, como, por exemplo, a matemática, a leitura, as ciências etc. Tendo o presente estudo incidido nas notas escolares de um conjunto alargado de disciplinas, não as analisou de forma individual, com a exceção do caso de português. Aqui, o resultado encontrado é curioso e parece ir ao encontro do que se acaba de afirmar, já que se encontraram relações não só com a componente cognitiva, mas também com a comportamental. A situação da dimensão afetiva, que não se mostrou associada a nenhuma área específica (disciplina de português e escrita), mas que está ligada com as outras variáveis (média das notas, repetências) reforça a necessidade de um estudo aprofundado sobre as relações preferenciais de cada uma das dimensões. Para além do já apontado, isto é, da possível ligação de cada uma das dimensões com áreas acadêmicas particulares, é desejável que se perceba, igualmente, se há uma diferenciação em função do grau de generalidade dos indicadores de desempenho considerados: em áreas específicas (como é o caso da escrita), em disciplinas (como, por exemplo, o português), e no geral (seja a média das notas obtidas em todas as disciplinas, seja o número de repetências).

A ausência de uma relação entre a dimensão agenciativa e qualquer indicador de desempenho acadêmico, incluindo o da escrita, levanta outro tipo de problemas. Algumas das razões já aduzidas podem ser aqui evocadas: a natureza dos itens do questionário e o fato de essa dimensão ser suscetível de estar associada a áreas escolares não contempladas 
no estudo. Mas poderá haver uma outra razão. Será que aquilo que é avaliado nessa dimensão, isto é, a capacidade de intervir proativamente e influenciar o percurso escolar, não remete a um tipo de comportamento não muito valorizado pelo nosso sistema de ensino? Os bons resultados acadêmicos poderão indiciar mais conformismo do que iniciativa? São questões que convém estudar no futuro, procurando perceber melhor o que caracteriza o bom aluno na escola atual.

Deve-se realçar, ainda, que, confirmando a investigação nesse domínio (BORKOWSKI; THORPE, 1994), o envolvimento, pelo menos nas suas dimensões afetiva e comportamental, encontrou-se associado ao nível socioeducativo dos alunos, mais concretamente ao das suas mães. 0 mesmo resultado não se verificou relativamente ao sexo, já que no presente estudo não foi encontrada nenhuma relação entre essa variável e o envolvimento.

Convém, por último, referir que as relações encontradas no nosso estudo não nos dizem nada acerca de uma questão essencial que é a do sentido da direção entre envolvimento e desempenho acadêmico. Embora tudo indique que essa relação é bidirecional (FINN; ROCK, 1997; LI, LERNER, LERNER, 2010), seria importante, através de estudos com design experimental, testar a eficácia de programas de diferentes tipos, incidindo uns no envolvimento e outros no ensino e na aprendizagem de áreas específicas. Os efeitos recíprocos de cada um desses programas ajudarão a esclarecer o sentido da relação entre as duas variáveis e a pensar as práticas pedagógicas.

Em suma, do estudo efetuado, pode concluir-se que o papel do envolvimento se viu amplamente confurmado no caso do desempenho acadêmico, ainda que não totalmente no que respeita à escrita. Do mesmo modo, nem todas as dimensões do envolvimento se mostraram igualmente relevantes, no que toca às suas relações com o desempenho escolar em geral e com a escrita em particular. Futuras investigações que ajudem a esclarecer a natureza dessas relações e que, através do recurso a designs experimentais, procurem perceber o sentido causal dessas mesmas relações serão muito oportunas e necessárias.

\section{Referências}

AINLEY, Mary. Styles of engagement with learning: multidimensional assessment of their relationship with strategy use and school achievement. Journal of Educational Psychology, Washington, DC, v. 85, n. 3, p. 395-405, set. 1993.

ANDERMAN, Eric; PATRICK, Helen. Achievement goal theory, conceptualization of ability/ intelligence, and classroom climate. In: CHRISTENSON, Sandra; RESCHLY, Amy; WYLIE, Cathy (Ed.). Handbook of research on student engagement. New York: Springer, 2012. p. 173-191.

APPLETON, James J.; CHRISTENSON, Sandra L.; FURLONG, Michael J. Student engagement with school: critical conceptual and methodological issues of the construct. Psychology in the Schools, Chichester, v. 45, n. 5, p. 369-386, maio 2008. Disponível em: <http://onlinelibrary.wiley.com/doi/10.1002/pits.20303/ abstract>. Acesso em: 12 mar. 2016.

APPLETON, James J. et al. Measuring cognitive and psychological engagement: Validation of the Student Engagement Instrument. Journal of School Psychology, Amsterdam, v. 44, n. 5, p. 427-445, out. 2006. 
BORKOWSKI, John; THORPE, Pamela. Life-span perspective on underachievement in self-regulation of learning and performance. In: SCHUNK, Dale; ZIMMERMAN, Barry (Ed.). Self-regulation of learning and performance: issues and educational applications. New Jersey: Lawrence Erlbaum Associates. 1994. p. 45-73.

BOSCOLO, Pietro; GELATI, Carmen. Best practices in promoting motivation for writing. In: GRAHAM, Steve; MACARTHUR, Charles; FITZGERAL, Jill (Ed.). Best practices in writing instruction. New York: Guilford, 2007. p. 202-221.

BOSCOLO, Pietro; HIDI, Suzanne. The multiple meanings of motivation to write. In: HIDI, Suzanne; BOSCOLO, Pietro (Ed.). Writing and motivation. Amsterdam: Elsevier, 2007. p. 1-14.

BRUNING; Roger; KAUFFMAN, Douglas. Self-efficacy beliefs and motivation in writing development. In: MacARTHUR, Charles; GRAHAM, Steve; FITZGERALD, Jill (Ed.). Handbook of writing research. 2. ed. New York: Guilford, 2016. p. 160-173.

CARAWAY, Kirsten, et al. Self-efficacy, goal orientation and fear of failure as predictors of school engagement in high school students. Psychology in the Schools, Chichester, v. 40, n. 4, p. 417-724, jul. 2003.

ECCLES, Jacquelynne S. Negative effects of traditional middle schools on students' motivation. Elementary School Journal, Chicago, v. 93, n. 5, p. 553-574, maio 1993.

ELLIOT, Andrew J. Approach and avoidance motivation and achievement goals. Educational Psychologist, Oxford, v. 34, n. 3, p. 169-189, jun. 1999.

FINN, Jeremy. Withdrawing from school. Review of Educational Research, Los Angeles, v. 59, n. 2, p. 117-142, jun. 1989.

FINN, Jeremy; ROCK, Donald. Academic success among students at risk for school failure. Journal of Applied Psychology, Washington, DC, v. 82, n. 2, p. 221-234, abr. 1997.

FREDRICKS, Jennifer A.; BLUMENFELD, Phyllis C.; PARIS, Alison H. School engagement: potential of the concept, state of the evidence. Review of Educational Research, Los Angeles, v. 74, n. 1, p. 59-109, mar. 2004.

GABINETE DE AVALIAÇÃO EDUCACIONAL. Provas finais de ciclo. Exames finais nacionais: relatório 2012. Lisboa: Instituto de Avaliação Educativa, 2013. 175 p.

GLANVILLE, Jennifer L.; WILDHAGEN, Tina. The measurement of school engagement: assessing dimensionality and measurement invariance across race and ethnicity. Educational and Psychological Measurement, Los Angeles, v. 67, n. 6, p. 1019-1041, jun. 2007.

GO0DENOW, Carol. The psychological sense of school membership among adolescents: scale development and educational correlates. Psychology in the Schools, Chichester, v. 30, n. 1, p. 79-90, jan. 1993.

GUTHRIE, John; WIGIELD, Allan. Engagement and motivation in Reading. In: KAMIL, Michael et al. (Ed.). Handbook of reading research. v. 3. Mahwah: Lawrence Erlbaum. 2000. p. 403-424. 
GUTMAN, Leslie; MIDGLEY, Carol. The role of protective factors in supporting the academic achievement of poor African American students during the middle school transition. Journal of Youth and Adolescence, New York, v. 29, n. 2, p. 223-249. abr. 2000.

HAYES, John R.; FLOWER, Linda S. Identifying the organisation of writing processes. In: GREGG, Lee; STEINBERG, Erwin (Ed.). Cognitive processes in writing. Hillsdale: Lawrence Erlbaum. 1980. p. 3-30.

HENRY, Kimberly; KNIGHT, Kelly; THORNBERRY, Terence. School disengagement as a predictor of dropout, delinquency, and problem substance use during adolescence and early adulthood. Journal of Youth and Adolescence, New York, v. 41, n. 2, p. 156-166. fev. 2012.

HIDI, Suzanne; BOSCOLO, Pietro (Ed.) Writing and motivation. Amsterdam: Elsevier, 2007. 334 p.

HIRSCHFIELD, Paul; GASPER, Joseph. The relationship between school engagement and delinquency in late childhood and early adolescence. Journal of Youth and Adolescence, New York, v. 40, n. 1, p. 3-22, jan. 2011.

JANOSZ, Michael et al. School engagement trajectories and their differential predictive relations to dropout. Journal of Social Issues, Chichester, v. 64, n. 1, p. 21-40, mar. 2008.

JIMERSON, Shane; CAMPOS, Emily; GREEN, Jennifer G. Towards an understanding of definitions and measures of student engagement in schools and related terms. The California School Psychologist, Sacramento, v. 8, n. 1. p. 7-27, 2003.

JOHNSON, Monica K.; CROSNOE, Robert; ELDER, Glen H. Students' attachment and academic engagement: the role of race and ethnicity. Sociology of Education, Los Angels, v. 74, n. 4, p. 318-340, out. 2001.

KELLOGG, Ronal. The psychology of writing. New York: Oxford University Press. 1994. 253 p.

KLEM, Adena; CONNELL, James. Relationships matter: linking teacher support to student engagement and achievement. Journal of School Health, Chichester, v. 74, n. 7, p. 264-274, set. 2004.

LANE, Kathleen et al. Teaching writing strategies to young students struggling with writing and at risk for behavioral disorders: self-regulated strategy development. Teaching Exceptional Children, Los Angeles, v. 39, n. 1, p. 60-64, set./out. 2006.

LI, Yibing et al. Out-of-school time activity participation, school engagement and positive youth development: findings from the 4-H study of positive youth development Tufts University. Journal of Youth Development, Raleigh, v. 3, n. 3, p. 1-16, 2008. Disponível em: <http://www.nae4ha.com/assets/documents/ JYD_09080303_final.pdf>. Article 080303FA001. Acesso em: 13 mai. 2017.

LI, Yibing; LERNER, Richard. Trajectories of school engagement during adolescence: implications for grades, depression, delinquency, and substance use. Developmental Psychology, Washington, DC, v. 47, n. 1, p. 233-247, jan. 2011.

LI, Yibing; LERNER, Jacqueline; LERNER Richard. Personal and ecological assets and academic competence in early adolescence: the mediating role of school engagement. Journal of Youth and Adolescence, New York, v. 39, n. 7, p. 801-815, jul. 2010. 
MACARTHUR, Charles; GRAHAM, Steve. Writing research from a cognitive perspective. In: MacARTHUR, Charles; GRAHAM, Steve; FITZGERALD, Jill (Ed.). Handbook of writing research 2. ed. New York: Guilford, 2016. p. 24-40.

MARTIN, Andrew J. Examining a multidimensional model of student motivation and engagement using a construct validation approach. British Journal of Educational Psychology, Chichester, v. 77, n. 2, p. 413440, jun. 2007.

NATIONAL CENTER FOR EDUCATION STATISTICS. The nation's report card: writing 2011. Washington, DC: Institute of Educational Sciences; U.S. Department of Education, 2012. 47 p.

NEWTON, Rae R.; Rudestam, Kjell E. Your statistical consultant: answers to your data analysis questions. 2. ed. Thousand Oaks: Sage, 2013. 356 p.

PAYNE, Allison; GOTTFREDSON, Denise; GOTTFREDSON, Gary. Schools as communities: the relationships among communal school organization, student bonding, and social disorder. Criminology, Chichester, v. 41, n. 3, p. 749-777, ago. 2003.

REEVE, Johnmarshall; TSENG, CHing-Mei. Agency as a fourth aspect of students' engagement during learning activities. Contemporary Educational Psychology. Amsterdam, v. 36, n. 4, p. 257-267, maio 2011.

REID, Ken. The causes, views, and traits of school absenteeism and truancy: an analytical review. Research in Education, Los Angeles, v. 74, p. 59-82, nov. 2005.

SCARDAMALIA, Marlene; BEREITER, Carl. Writing. In: DILLON, Ronna; STERNBERg, Robert (Ed.). Cognition and instruction. Orlando: Academic Press, 1986. p. 59-81.

SHARP, Caroline; KEYS, Wendy; BENEFIELD, Pauline. Homework: a review of recent research. Slough: National Foundation for Educational Research (NFER), 2001. 62 p.

SIMON-MORTON, Bruce G.; CHEN, Rusan. Peer and parent influences on school engagement among early adolescents. Youth \& Society. Los Angeles, v. 41, n. 1, p. 3-25, set. 2009.

SKINNER, Ellen A.; BELMONT, Michael J. Motivation in the classroom: reciprocal effects of teacher behavior and student engagement across the school year. Journal of Educational Psychology, Washington, DC, v. 85, n. 4, p. 571-581, dec. 1993.

SKINNER, Ellen, A. et al. Engagement and disaffection in the classroom: part of a larger motivational dynamic? Journal of Educational Psychology, Washington, DC, v. 100, n. 4, p. 765-781, nov. 2008.

SKINNER, Ellen, A. et al. Engagement and disaffection as organizational constructs in the dynamics of motivational developmental. In: WENTZEL, Kathryn; WIGFIELD, Allan (Ed.). Handbook of motivation in school. Mahwah: Erlbaum. 2009. p. 223-245.

VAN EEMEREN, Frans; GROOTENDORST, Rob. A systematic theory of argumentation: the pragma-dialectical approach. Cambridge: Cambridge University Press, 2004. 216 p. 
VEIGA, Feliciano, H. Envolvimento dos alunos na escola: elaboração de uma nova escala de avaliação. International Journal of Developmental and Educational Psychology, Badajoz, v. 1, n. 1, p. 441 - 450, 2013.

VEIGA, Feliciano, H. Assessing student engagement in school: development and validation of a fourdimensional scale. Procedia - Social and Behavioral Sciences, Amsterdam, v. 217, p. 813-819, fev. 2016. http://dx.doi.org/10.1016/j.sbspro.2016.02.153529-543.

VEIGA, Feliciano, H. et al. Envolvimento dos alunos na escola: conceito e relação com o desempenho académico - sua importância na formação de professores. Revista Portuguesa de Pedagogia, Coimbra, v. 46, n. 2, p. 31-47, jul. 2012.

VOELKL, Kristin E. Identification with school. American Journal of Education, Chicago, v. 105, n. 3, p. 294318, maio. 1997.

WANG, Ming-Te; HOLCOMBE, Rebecca. Adolescents' perceptions of school environment, engagement, and academic achievement in middle school. American Educational Research Journal, Los Angeles, v. 47, n. 3, p. 633-662, set. 2010.

WIGFIELD, Allan; ECCLES, Jacquelynne S. Expectancy - value theory of motivation. Contemporary Educational Psychology, Amsterdam, v. 25, n. 1, p. 68-81, jan. 2000.

Recebido em: 01.08.2017

Revisões em: 01.12.2017

Aprovado em: 06.02.2018

Maria Isabel Festas é professora catedrática na Faculdade de Psicologia e de Ciências da Educação da Universidade de Coimbra, onde é coordenadora do doutoramento em ciências da educação. É investigadora do Centro de Estudos Interdisciplinares do Século XX (CEIS20) da Universidade de Coimbra.

Maria José Prata é estudante de doutoramento em ciências da educação na Faculdade de Psicologia e de Ciências da Educação da Universidade de Coimbra e investigadora do Centro de Estudos Interdisciplinares do Século XX (CEIS20) da Universidade de Coimbra.

Albertina Lima de Oliveira é professora auxiliar na Faculdade de Psicologia e de Ciências da Educação da Universidade de Coimbra, onde é membro da Comissão de Ética e Deontologia da Investigação. É investigadora do Centro de Estudos Interdisciplinares do Século XX (CEIS20) da Universidade de Coimbra.

Feliciano Veiga é professor catedrático no Instituto de Educação da Universidade de Lisboa, onde é coordenador do doutoramento em psicologia da educação, membro do Conselho Científico e presidente da Comissão de Ética. É membro de júris de avaliação da FCT e da A3Es. 